\title{
Effectiveness and cerebral responses of multi-points acupuncture for primary insomnia: a preliminary randomized clinical trial and fMRI study
}

Yu-Kai Wang ${ }^{1}$, Tie Li ${ }^{2}$, Li-Juan Ha ${ }^{2}$, Zhong-Wen Lv' ${ }^{3}$, Fu-Chun Wang ${ }^{2,4}$, Zhi-Hong Wang ${ }^{2,4}$, Jing Mang ${ }^{1}$ and Zhong-Xin $\mathrm{Xu}^{1 *}$ (D)

\begin{abstract}
Background: Primary insomnia (PI) is characterized by difficulties in initiating sleep or maintaining sleep, which lead to many serious diseases. Acupuncture for PI has drawn attention with its effectiveness and safety. However, the operation of choosing acupoints lacks scientific suggestion. Our trial aims to provide reference and scientific basis for the selection of acupoints and to explore its possible mechanism.

Methods: A patient-assessor-blinded, randomized and sham controlled trial was designed to compare the efficacy of 5-weeks acupuncture at a single acupoint, the combination of multi-acupoints, and a sham point. The Pittsburgh sleep quality index and Athens Insomnia Scale questionnaire were used for the primary clinical outcomes, while polysomnography was performed for the secondary clinical outcomes. The resting state functional MRI was employed to detect the cerebral responses to acupuncture. The brain activity in resting state was measured by calculating the fractional amplitude of low-frequency fluctuations (fALFF), which reflected the idiopathic activity level of neurons in the resting state. These results were analyzed by two factorial ANOVA test and post-hoc t-tests.

Results: The clinical outcomes suggest that acupuncture could improve clinical symptoms, and the combination of multi-acupoints might lead to a better clinical efficacy. The rs-fMRI results suggested that the brain activity of certain regions was related to the sleep experience, and acupuncture could regulate the activity of these regions. Furthermore, the combination of multi-acupoints could impact more regions which were influenced by the sleep experience.
\end{abstract}

Conclusions: Acupuncture has been proven to be beneficial for PI patients, and the combination of multiacupoints might improve its efficacy.

Trial registration: This trial has been registered on the U.S. National Library of Medicine (https://clinicaltrials.gov) ClinicalTrials.gov Identifier: NCT02448602. Registered date: 14/04/2015.

Keywords: Primary insomnia, Acupuncture, Functional magnetic resonance imaging, Polysomnography

\footnotetext{
* Correspondence: xuzhongxin9999@sina.com

'Department of Neurology, China-Japan Union Hospital of Jilin University, Changchun, Jilin 130033, P.R. China

Full list of author information is available at the end of the article
} 


\section{Background}

Primary insomnia (PI) is defined as a symptom of prolonged sleep latency and difficulty in maintaining sleep [1]. As a prevalent sleep disorder in the global population with rising prevalence [2], PI has caused an increased incidence of serious medical conditions, such as obesity, heart disease, high blood pressure and diabetes [3]. In general, treatments for PI include nonpharmacologic and pharmacologic therapies. However, pharmacological therapies remain limited due to potential risks and barriers, including long-term usage and the range of undesirable side-effects [4]. As a result, the exploration for more convenient treatments with less side-effect for PI patients has continued for decades.

Acupuncture, which is a technique of inserting needles into particular points of body, has been proven to be effective for insomnia with minor side-effects $[5,6]$. Hence, acupuncture has been increasingly used in private clinics located in 46 states in the United States as a treatment for insomnia [7]. However, there is no standard regimen for acupuncture in the clinical treatment of insomnia, especially in the selection of acupoints. In general, acupuncturists could choose single acupoint or a combination of multi-acupoints with the same efficacy to treat insomnia. Although this is the main factor that affects efficacy, it remains unclear how the number of stimuli acupoints interfere with clinical efficacy. Furthermore, the mechanism of different efficacy produced by different acupuncture scheme remained unclear. Previous studies on the mechanism of acupuncture have revealed that acupuncture can affect the activation in brain regions through needling/tactile somatosensory specific stimuli [8-10]. Further, acupuncture might have lasting and strong sustained effects on cerebral functional regions, which was founded in a study, that is, the activation in brain regions at $15 \mathrm{~min}$ after acupuncture stimuli were greater than the activation at $5 \mathrm{~min}$ after acupuncture stimuli [11].

In order to determine which kind of acupoints scheme (a single acupoint or a combination of multi-acupoints) could produce better efficacy, a patient-assessor-blinded, randomized and sham controlled trial was designed for PI patients. In the present study, PI patients randomly accepted electro-acupuncture stimulation on single acupoint, multi-acupoints, sham points for 5 weeks. Pittsburgh sleep quality index (PSQI), Athens Insomnia Scale (AIS) questionnaires and the polysomnography (PSG) were performed to evaluate the sleep condition at baseline and after the treatment. Resting state functional magnetic resonance imaging (rs-fMRI) could detect blood oxygenation level dependent (BOLD) signals in brain in resting state [12]. Amplitude of low-frequency fluctuations (ALFF) could directly reflect idiopathic activity levels of neurons in the voxels according to the
BOLD signals [13]. Furthermore, a modified calculation called fractional amplitude of low-frequency fluctuation (fALFF), which means the ratio of the power spectrum of low frequency $(0.01-0.08 \mathrm{~Hz})$ to that of the entire frequency range, was developed to suppress non-specific noise components [14]. Taken together, the present study aims to provide a reference and scientific basis for the choice of acupoints in clinical treatment, and expect to be helpful in improving the clinical efficacy of acupuncture for PI patients.

\section{Methods \\ Subjects}

The present study is a patient-assessor-blinded, randomized and sham controlled trial (No. NCT02448602, registered on 14/04/2015). From September 2015 to September 2018, PI patients were recruited from outpatient clinics in the Neurological Department of ChinaJapan Union Hospital of Jilin University and Changchun University of Chinese Medicine. All subjects satisfied the following criteria. Inclusion criteria [15]: (1) patients who were over 18 years old, but under 65 years old; (2) patients who had no problems with communication and intelligence; (3) patients with a wake after sleep onset (WASO) or sleep onset latency (SOL) of > $30 \mathrm{~min}$ for at least 3 nights every week, and symptoms lasting for over 3 months; (3) patients with an AIS score of $\geq 6$; (4) patients with a PSQI score of $>7$. Exclusion criteria [15]: (1) patients with uncontrolled medical conditions suspected to interfere with sleep; (2) patients with uncontrolled psychiatric conditions requiring immediate treatment; (3) patients diagnosed of comorbid sleep disorders; (4) patients with a self-rating depression scale (SDS) or self-rating anxiety scale (SAS) of $\geq 50$; (5) patients with alcohol and/or other drug abuse or dependence; (6) Pregnant or nursing patients, or those of childbearing age who have not adopted appropriate birth control methods; (7) patients with who took hypnotic or sedating medications, or accepted acupuncture treatment in the recent 1 month.

The participants were informed that the intervention in this trial included three different acupuncture regimens (but they did not know the acupoints of other regimens except the regimens they accepted in the course of the trial), the treatment efficacy might be different, the patients would randomly accept one acupuncture regimens. Patients were included in the trial after approval and signature of informed consent (Permission number: CCZYFYLL2014-043).

\section{Acupuncture procedures}

The acupoints used in this trial are Shenmen (HT-7), Sanyinjiao (SP-6), Baihui (GV-20), which are recommended points for insomnia treatment $[5,6,16]$. HT-7 
is the point with the highest frequency of use [17], SP-6 and GV-20 are often used with HT-7 as combination regimen according to the Traditional Chinese Medicine (TCM) theory and clinical experience of acupuncture therapists [18]. Also, a sham point used as control group to exclude the placebo effect of acupuncture. The patients were randomly divided into three groups, and accepted electro-acupuncture for 5-weeks on different acupoints: single acupoints group (S-Acu group, bilateral HT-7) (Fig. 1a); multi-acupoints group (M-Acu group, combination of bilateral HT-7, bilateral SP-6 and single GV-20) (Fig. 1a-c); Non-acupoint group (N-Acu group, bilateral sham point at the junction point between the biceps brachii muscle and deltoid muscle) (Fig. 1d). The randomized achieve through the network system of Clinical Evaluation Center of China Academy of Chinese Medical Sciences. During the acupuncture, a $25 \times 0.35$ $\mathrm{mm}$ sterile and reusable acupuncture silver needles was inserted into the bilateral points at a depth of $10 \mathrm{~mm}$ [18] (the GV-20 was at a depth of 5-8 mm [19]). Another silver needle was inserting at a distance of $1 \mathrm{~cm}$ from the acupoint. The handle of these two needles were connected to an electro-acupuncture machine (Suzhou Medical Appliance Factory, China) with a frequency of $4 \mathrm{~Hz}[20]$ and an intensity that maintained the De-Qi sensation [21]. The Massachusetts General Hospital acupuncture sensation scales (MASS) was used to determine whether the De-Qi sensations was produced during the acupuncture. The intensity of soreness, numbness, heaviness, warmth, cold, sharp pain and dull pain were rate on a numerical scale of $1-10$ to screen patients with the De-Qi sensation. The acupuncture treatment lasted for 5 weeks, five times per week, and 30 min each time.

\section{Observation of clinical efficacy}

All patients accomplished the AIS and PSQI questionnaires at baseline and at the end of the 5-week treatment. The AIS questionnaire is a self-administered psychometric instrument that consisted of eight items. Each item was rated from 0 to 3 , for a total score range of 0-24. A score of "0" indicates no problem at all, while a score of "24" indicates very serious problems in all areas [22]. The PSQI questionnaire consists of 19 selfrated questions, which are grouped into seven component scores, each ranging from 0 to 3 . Then, the seven component scores are summed to yield a global PSQI score, which has a range of $0-21$, with higher scores indicating worse sleep quality [23].

Patients underwent the polysomnography (PSG, Embletta X100) assessment at baseline and the end of the 5-weeks treatment. The PSG test was performed according to American Academy of Sleep Medicine (AASM) standards after an adaption night in single rooms. The duration of the PSG recording was $480 \mathrm{~min}$. The PSG monitor physiologic data during the sleep cycle included the electrooculogram (EOG), electroencephalogram (EEG), electrocardiogram (ECG), electromyogram

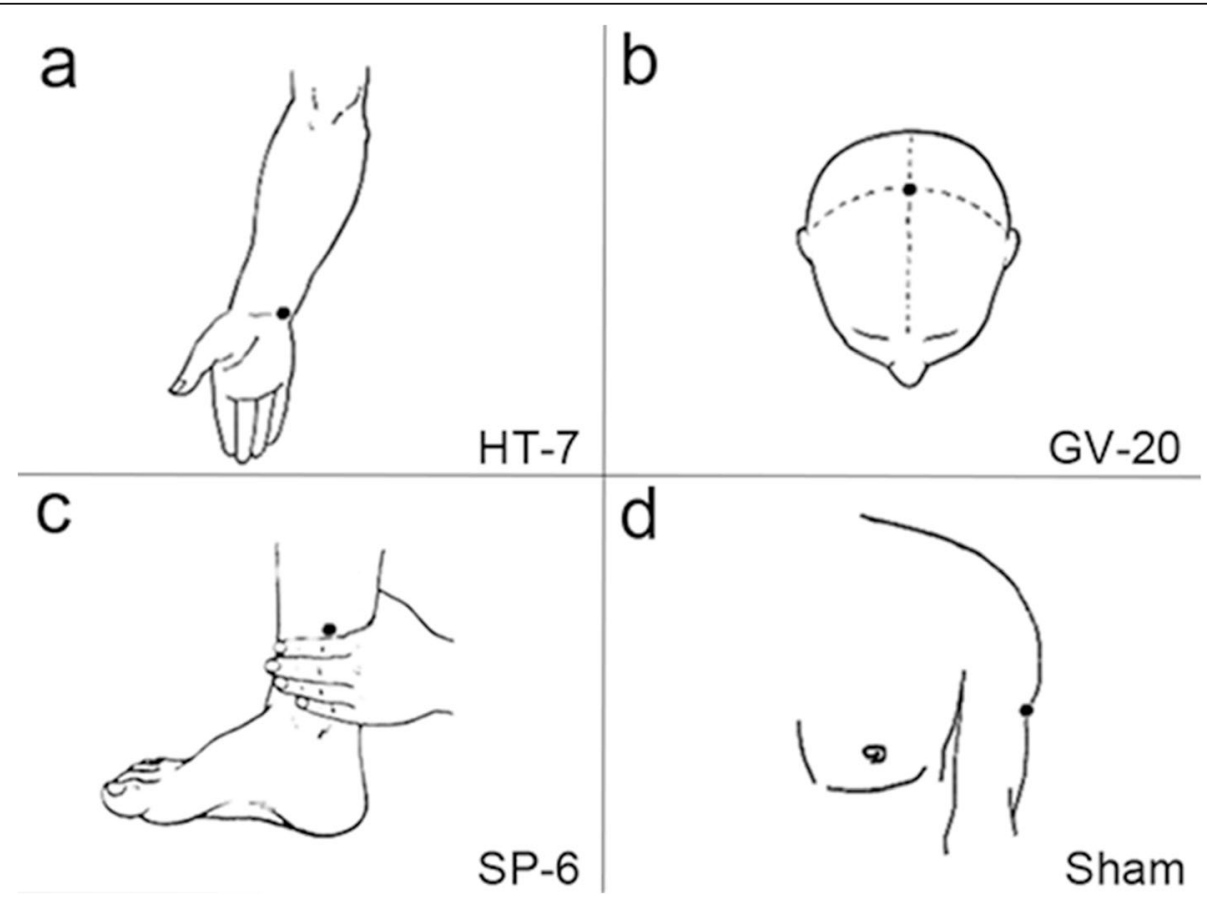

Fig. 1 The location of the acupoints and diagrammatic sketch of the fMRI scan process. a The location of the acupoint in S-Acu group. a-c The location of acupoints in M-Acu group. $\mathbf{d}$ The location of the sham point in N-Acu group 
(EMG) (submental and bilateral masseter), airflow measurements using both oronasal-thermal sensors and nasal air pressure transducers, rib cage and abdominal movement by inductance plethysmography using thoracoabdominal belts, and continuous pulse oximetry. Variables, including total sleep time (TST), sleep efficient (SE), sleep stages (stage NREM1 (N1), stage N2, stage N3, and stage REM), wake after sleep onset (WASO) and arousal index (AI), were analyzed with the abovementioned PSG data.

\section{Statistical analysis of clinical outcomes}

The clinical data were analyzed using SPSS 18.0 statistical software. The demographic characteristic of three groups including gender, age, SAS and SDS were analyzed with one-way ANOVA test. The MASS scale of three groups were analyzed with one-way ANOVA test. The primary and secondary clinical outcomes, the AIS / PSQI scores and the PSG data at baseline and after the treatment, were analyzed with a two factorial ANOVA testing. The change of clinical outcomes among three group were analyzed with ANOVA analyses and posthoc t-tests. All data were expressed as mean \pm standard deviation.

\section{FMRI data acquisition}

We stratified the patients in the S-Acu group who agreed to accept fMRI examination according to the age of $18 \sim 35,36 \sim 50,51 \sim 65$, and randomly selected 5 patients from each age group, a total of 15 patients received fMRI examination. The same were the M-Acu group and the N-Acu group. PI patients received rsfMRI assessment at baseline and 1 day after 5 weeks of acupuncture treatment between 8:00 a.m. and 10:00 a.m. during which the subjects were relatively awake.

The fMRI scan was completed on a $3.0 \mathrm{~T}$ whole-body MRI scanner (MAGNETOM-Skyra-SIEMENS) with a volume transmit head coil and 32-channel receive coil. The MRI sequences included the following: (1) T1-weighted MRI: data were acquired using a magnetization-prepared rapid gradient-echo sequence with TR/TE at $700 \mathrm{~ms} / 11$ $\mathrm{ms}$, FOV at $256 \times 256 \times 192$, and a voxel size of $1 \times 1 \times 0.9$; (2) rs-fMRI: data were acquired using an echo planar imaging (EPI) sequence sensitive to BOLD with TR/TE/FA at $2020 \mathrm{~ms} / 30 \mathrm{~ms} / 90^{\circ}$, and FOV at $106 \times 106 \times 46$, and a voxel size of $2.4 \times 2.4 \times 3.75$. The rs-fMRI scan duration lasted 200 TR [15].

\section{Rs-fMRI data processing}

The main preprocessing steps of rs-fMRI included the following [15]: Digital Imaging and Communications in Medicine (DICOM) data were converted into NIFTI data; the first 10 volumes were discarded for signal stabilization and subject adaptation; then, slice timing, spatial realignment, head motion correction, individual registration between high-resolution $\mathrm{T} 1$ and echo planar imaging (EPI) images, T1 segmentation with the Diffeomorphic Anatomical Registration Through Exponentiated Lie algebra (DARTEL) and spatial normalization to register rs-fMRI data sets to the Montreal Neurological Institute (MNI) space were performed, along with resampling to $3 \times 3 \times 3 \mathrm{~mm}^{3}$ cube voxels; and head motion estimation, $>1.5 \mathrm{~mm}$ of the maximal translation or $1.5^{\circ}$ of the maximal rotation, was excluded from the final analysis. Then the normalized data were spatially smoothed using a $6 \mathrm{~mm}$ full-width half-maximum Gaussian kernel. Linear detrending and nuisance linear regression (including the white matter, the cerebrospinal fluid and head motion parameters) were performed, and a temporal bandpass filter $(0.01-0.08 \mathrm{~Hz})$ was applied to reduce the effects of head motion and nonneuronal BOLD fluctuations [24]. ALFF and fALFF were calculated with DPARSF package for each subject [14].

This preprocessing was performed using the Data Processing Assistant for Resting-State fMRI (DPARSF) package (http://www.restfmri.net) [24] based on statistical parametric mapping (SPM8, Welcome Department of Imaging Neuroscience, Institute of Neurology, London; http://www.fil.ion.ucl.ac.uk/spm), which was run on MATLAB (MathWorks, Natick, MA, USA).

\section{Rs-fMRI data analysis}

SPM8 was employed for the rs-fMRI data analysis. First, the relationship between the clinical outcome and brain activity were studied. The fALFF value of PI patients was analyzed by multiple regression analysis with AIS score as covariable $(P<0.001$, FWE correct $P=0.05$, cluster size $>30$ ).

The altered fALFF value after treatment of three groups were analyzed by one-way ANOVA with-in subject test (flexible factorial model) with the groups (S-Acu group, M-Acu group, N-Acu group) as the between-subject factor and the repeated measure (baseline and after treatment) as the within-subjects factor $(P<0.001$, FWE correct $P=0.05$, cluster size $>$ 30). Furthermore, the difference of S-Acu group vs $\mathrm{N}$-Acu group, and S-Acu group vs M-Acu group were analyzed with post-hoc t-tests $(P<0.005$, FWE correct $P=0.05$, cluster size $>30$ ). All results were presented by the REST v1.8 software [25].

\section{Patient safety}

Any adverse events related to the acupuncture treatment, including unfavorable or unintended signs, symptoms, or diseases occurring after treatment, were observed and reported. The patients will immediately terminate the trial if adverse event happened. 


\section{Quality control}

One investigator and an acupuncturist participated in the present study, and they both received special training before the trial, in order to ensure consistency in practice. The training program included the following: diagnosis, inclusion and exclusion criteria, case reports, location of the acupoints, and acupuncture manipulation techniques. The investigator did not know the groupings, the group assigned for each patient, and the person responsible for the evaluation of patients and analysis of all data before and after the acupuncture treatment. The acupuncturist, who has been engaged in clinical acupuncture for more than 3 years, was responsible for the administration of treatment and the operation of the acupuncture. All test results were reviewed and adjudicated by a professional neurologist, who did not know which group the patient's result belonged to, or who responsible for the evaluation of clinical data. The patients were not informed of the treatment for other groups, except for the treatment they were receiving. The accuracy and quality of the whole study was ensured through regular monitoring during the intervention periods.

\section{Results}

\section{Demographic characteristic in different groups}

In our trials, 129 patients (mean age: $52.66 \pm 11.14$ years old, 27 males) were included in the final analysis. The subjects were randomly divided into 3 groups (S-Acu group: $n=43$; M-Acu group: $n=43$; N-Acu group: $n=$ 43). There were no significant differences in gender, age, PSQI, AIS, SAS and SDS scores among the three groups $(P>0.05)$ (Table 1$)$. And the type of needling sensations was recorded with MASS scale. There were no significant differences among the intensity of seven sensations $(P>0.05)$ (Table 2).

Table 1 Demographic characteristic at baseline in different groups

\begin{tabular}{llll}
\hline Parameter & S-Acu group (HT-7) & $\begin{array}{l}\text { M-Acu group (HT-7, } \\
\text { GV-20 and SP-6) }\end{array}$ & $\begin{array}{l}\text { N-Acu group } \\
\text { (sham point) }\end{array}$ \\
\hline Sex & & & 7 \\
Male & 11 & 9 & 36 \\
Female & 32 & 34 & $51.10 \pm 11.60$ \\
Age(years) & $55.42 \pm 9.23$ & $51.45 \pm 12.10$ & $15.21 \pm 2.95$ \\
PSQI & $14.70 \pm 2.99$ & $14.84 \pm 2.77$ & $13.56 \pm 4.48$ \\
AIS & $12.40 \pm 3.14$ & $13.26 \pm 4.21$ & $38.56 \pm 7.40$ \\
SAS & $38.95 \pm 3.96$ & $40.19 \pm 5.75$ & $34.70 \pm 8.59$ \\
\hline SDS & $36.86 \pm 7.01$ & $36.12 \pm 6.72$ &
\end{tabular}

PSQI Pittsburgh sleep Quality index, AIS Athens Insomnia Scale, SAS self-rating anxiety scale, SDS self-rating depression scale, $S$-Acu group single acupoint group, M-Acu group multi-acupoints group, N-Acu group non-acupoint group
Table 2 Comparisons of the intensity of De-Qi sensations among the three groups

\begin{tabular}{lllll}
\hline Sensations & $\begin{array}{l}\text { S-Acu group } \\
\text { (HT-7) }\end{array}$ & $\begin{array}{l}\text { M-Acu group (HT-7, } \\
\text { GV-20 and SP-6) }\end{array}$ & $\begin{array}{l}\text { N-Acu group } \\
\text { (sham point) }\end{array}$ & $P$ \\
\hline soreness & $1.74 \pm 0.76$ & $2.02 \pm 0.77$ & $1.93 \pm 0.74$ & 0.223 \\
numbness & $1.74 \pm 0.69$ & $1.91 \pm 0.68$ & $1.79 \pm 0.68$ & 0.526 \\
heaviness & $2.74 \pm 0.76$ & $2.98 \pm 0.80$ & $2.77 \pm 0.78$ & 0.318 \\
warmth & $1.51 \pm 0.74$ & $1.58 \pm 0.79$ & $1.47 \pm 0.74$ & 0.773 \\
cold & $0.74 \pm 0.76$ & $0.63 \pm 0.72$ & $0.70 \pm 0.74$ & 0.766 \\
sharp pain & $2.74 \pm 0.76$ & $2.93 \pm 0.80$ & $2.86 \pm 0.74$ & 0.526 \\
dull pain & $2.56 \pm 0.67$ & $2.65 \pm 0.65$ & $2.58 \pm 0.66$ & 0.794 \\
\hline
\end{tabular}

S-Acu group single acupoint group, $M$-Acu group multi-acupoints group, $N$-Acu group non-acupoint group

\section{Primary clinical outcomes in different groups}

After the 5-week acupuncture treatment, the AIS score and PSQI score were significantly decreased in all groups $(P<0.001)$. There were significant differences in AIS $(P=0.025) /$ PSQI scores $(P=0.001)$ among the three groups after acupuncture treatment. So, one-way ANOVA and post-hoc t-tests were performed for the decreased AIS and PSQI score of three groups. The decreased AIS score of M-Acu group was significantly higher than that of $\mathrm{N}$-Acu group $(P=0.026)$. And the decreased PSQI score of S-Acu group $(P=0.009)$ and M-Acu group $(P=0.004)$ were significantly higher than that of N-Acu group (Table 3).

\section{Secondary clinical outcomes in the different groups}

At baseline, there were no significant difference in the WASO $(P=0.448)$, TST $(P=0.271)$, SE $(P=0.381)$, AI $(P=0.055), \mathrm{N} 1 \quad(P=0.305), \mathrm{N} 2 \quad(P=0.368), \mathrm{N} 3 \quad(P=$ $0.373), \mathrm{R}(P=0.890)$ among three groups. After 5 -weeks acupuncture, the TST $(P=0.020), \mathrm{R}(P=0.001)$ of SAcu group were significant changed. The WASO $(P=$ $0.004)$, TST $(P<0.001)$, SE $(P=0.001)$, AI $(P=0.009)$,

Table 3 The AIS and PSQI scores at baseline and after acupuncture treatment in different groups

\begin{tabular}{llll}
\hline & $\begin{array}{l}\text { S-Acu group } \\
(\mathrm{HT}-7)\end{array}$ & $\begin{array}{l}\text { M-Acu group (HT-7, } \\
\text { GV-20 and SP-6) }\end{array}$ & $\begin{array}{l}\text { N-Acu group } \\
\text { (sham point) }\end{array}$ \\
\hline AIS & $12.40 \pm 3.14$ & $13.26 \pm 4.21$ & $13.56 \pm 4.48$ \\
Baseline & $7.00 \pm 3.90^{\mathrm{a}}$ & $5.91 \pm 3.92^{\mathrm{a}}$ & $8.88 \pm 3.36^{\mathrm{a}}$ \\
Post-treatment & $5.40 \pm 4.63$ & $7.35 \pm 3.85^{\mathrm{b}}$ & $4.67 \pm 4.52$ \\
Decrease & & & \\
& & PSQI & $15.21 \pm 2.95$ \\
Baseline & $14.70 \pm 2.99$ & $14.84 \pm 2.77$ & $11.60 \pm 3.54^{\mathrm{a}}$ \\
Post-treatment & $8.79 \pm 3.18^{\mathrm{a}}$ & $8.49 \pm 3.36^{\mathrm{a}}$ & $3.60 \pm 3.63$ \\
Decrease & $5.91 \pm 3.03^{\mathrm{c}}$ & $6.35 \pm 3.39^{\mathrm{c}}$ & \\
\hline
\end{tabular}

${ }^{\mathrm{a}} P<0.01$ (compared with baseline); ${ }^{\mathrm{b}} \mathrm{P}<0.05$ (compared with $\mathrm{N}$-Acu group); ${ }^{c} P<0.01$ (compared with $\mathrm{N}$-Acu group). $\mathrm{S}$-Acu group single acupoint group, $M$ Acu group multi-acupoints group, $\mathrm{N}-\mathrm{Acu}$ group non-acupoint group 
Table 4 The PSG data at baseline and after acupuncture treatment in different groups

\begin{tabular}{|c|c|c|c|c|c|c|}
\hline & \multicolumn{2}{|l|}{ S-Acu group } & \multicolumn{2}{|l|}{ M-Acu group } & \multicolumn{2}{|l|}{ N-Acu group } \\
\hline & Baseline & Post-treatment & Baseline & Post-treatment & Baseline & Post-treatment \\
\hline WASO (minutes) & $198.58 \pm 89.64$ & $179.06 \pm 76.58$ & $203.57 \pm 73.54$ & $154.84 \pm 91.19^{\mathrm{a}}$ & $214.24 \pm 68.41$ & $228.27 \pm 67.80$ \\
\hline TST (minutes) & $248.05 \pm 91.77$ & $288.25 \pm 76.51^{b}$ & $247.50 \pm 76.03$ & $319.90 \pm 92.51^{a}$ & $236.80 \pm 72.25$ & $247.56 \pm 66.50$ \\
\hline SE (\%) & $55.44 \pm 20.39$ & $61.70 \pm 16.25$ & $54.79 \pm 16.66$ & $67.34 \pm 19.33^{a}$ & $52.37 \pm 15.53$ & $52.06 \pm 14.12$ \\
\hline $\mathrm{Al}$ & $27.12 \pm 12.72$ & $21.51 \pm 11.01$ & $27.58 \pm 10.77$ & $21.16 \pm 9.71^{\mathrm{a}}$ & $26.77 \pm 10.34$ & $22.86 \pm 11.57$ \\
\hline N1 (minutes) & $40.80 \pm 30.49$ & $38.41 \pm 22.61$ & $36.94 \pm 20.67$ & $38.27 \pm 24.70$ & $37.49 \pm 25.97$ & $35.37 \pm 23.34$ \\
\hline N2 (minutes) & $62.21 \pm 49.88$ & $60.64 \pm 52.78$ & $60.53 \pm 63.38$ & $76.99 \pm 68.21$ & $52.45 \pm 53.97$ & $58.77 \pm 47.42$ \\
\hline N3 (minutes) & $74.99 \pm 48.69$ & $85.64 \pm 48.11$ & $66.98 \pm 45.07$ & $98.26 \pm 47.43^{a}$ & $69.80 \pm 36.80$ & $63.08 \pm 33.05$ \\
\hline $\mathrm{R}$ (minutes) & $70.05 \pm 45.01$ & $103.56 \pm 44.18^{a}$ & $83.05 \pm 52.23$ & $106.38 \pm 44.79^{b}$ & $77.06 \pm 41.47$ & $90.34 \pm 42.93$ \\
\hline
\end{tabular}

${ }^{\mathrm{a}} P<0.01$ (compared with baseline); ${ }^{\mathrm{b}} P<0.05$ (compared with baseline); WASO wake after sleep onset, TST total sleep time, SE sleep efficient, $A I$ arousal index, $N 1$ first stage of non-rapid eye movement sleep, N2 second stage of non-rapid eye movement sleep, $N 3$ third stage of non-rapid eye movement sleep, $R$ sleep stage of rapid eye movement, S-Acu group single acupoint group, $M$-Acu group multi-acupoints group, $N$-Acu group non-acupoint group

N3 $(P=0.001), \mathrm{R}(P=0.017)$ of $\mathrm{M}-\mathrm{Acu}$ group were significant changed. However, there was no significant difference was found in N-Acu group after 5-weeks acupuncture. There were significant differences in the WASO $(P<0.001) /$ TST $(P<0.001) / \mathrm{SE}(P<0.001) /$ N3 $(P=0.001)$ among three groups after acupuncture treatment (Table 4). So, the change of WASO, TST, SE, $\mathrm{N} 3$ in S-Acu group and M-Acu group were compared using independent two sample T-test. The results showed that the increase of TST in M-Acu group was significantly higher than that in S-Acu group $(P=$ 0.042). The decrease of WASO and the increase of SE and N3 in M-Acu group were higher than those in SAcu group, but there was no significant difference (Table 5).

\section{Rs-fMRI results in different groups}

\section{The relationship between the fALFF value of brain regions and AIS scores of PI}

We found a negative correlation between fALFF and AIS scores in the following brain regions: right cerebellum posterior lobe, temporal gyrus (left middle temporal gyrus, left superior temporal gyrus), left extranuclear, right anterior cingulate, left parahippocampa gyrus, frontal gyrus (bilateral inferior frontal gyrus, left middle frontal gyrus, bilateral superior frontal gyrus),

Table 5 The comparison of significant varied PSG data between S-Acu group and M-Acu group

\begin{tabular}{lll}
\hline & $\begin{array}{l}\text { S-Acu group } \\
\text { (HT-7) }\end{array}$ & $\begin{array}{l}\text { M-Acu group (HT-7, } \\
\text { GV-20 and SP-6) }\end{array}$ \\
\hline The increase of TST (minutes) & $40.20 \pm 55.48$ & $72.40 \pm 85.74^{\mathrm{a}}$ \\
The increase of SE (\%) & $6.26 \pm 12.44$ & $12.55 \pm 18.65$ \\
The increase of N3 (minutes) & $10.65 \pm 48.61$ & $31.28 \pm 50.45$ \\
The decrease of WASO (minutes) & $19.53 \pm 54.19$ & $48.73 \pm 87.83$ \\
\hline
\end{tabular}

${ }^{a} P<0.05$ (compared with S-Acu group); TST total sleep time, SE sleep efficient, $N 3$ third stage of non-rapid eye movement sleep, WASO wake after sleep onset, S-Acu group single acupoint group, $M$-Acu group multi-acupoints group parietal lobule (right precentral gyrus, left postcentral gyrus, left supramarginal gyrus, left inferior parietal lobule, left superior parietal lobule, left precuneus) $(P<0.001$, FWE correct $P=0.05$, cluster size $>30)$ (Table 6).

Table 6 The relationship between the fALFF value of brain regions and AIS scores of PI

\begin{tabular}{|c|c|c|c|c|c|c|c|}
\hline \multirow[t]{2}{*}{ Brain regions } & \multirow[t]{2}{*}{$\mathrm{BA}$} & \multirow[t]{2}{*}{ Side } & \multirow{2}{*}{$\begin{array}{l}\text { Cluster } \\
\text { size }\end{array}$} & \multicolumn{3}{|l|}{$\mathrm{MNI}$} & \multirow{2}{*}{$\begin{array}{l}\text { t- } \\
\text { value }\end{array}$} \\
\hline & & & & $x$ & Y & Z & \\
\hline Cerebellum Posterior Lobe & & $\mathrm{R}$ & 37 & 9 & -42 & -60 & -5.42 \\
\hline Middle Temporal Gyrus & & $L$ & 34 & -57 & -57 & -3 & -6.25 \\
\hline Middle Temporal Gyrus & 22 & $L$ & 217 & -57 & -42 & 0 & -7.53 \\
\hline \multicolumn{8}{|l|}{ Superior Temporal Gyrus } \\
\hline Parahippocampa Gyrus & & $\mathrm{L}$ & 36 & -24 & -27 & -27 & -5.56 \\
\hline Extra-Nuclear & & $L$ & 79 & -21 & 27 & 0 & -5.67 \\
\hline Anterior Cingulate & & $\mathrm{R}$ & 345 & 9 & 30 & 18 & -5.81 \\
\hline \multicolumn{8}{|l|}{ Inferior Frontal Gyrus } \\
\hline Middle Frontal Gyrus & \multirow{3}{*}{$\begin{array}{l}9 \\
6\end{array}$} & \multirow{3}{*}{$\mathrm{L}$} & \multirow[t]{3}{*}{1599} & \multirow[t]{3}{*}{-24} & \multirow[t]{3}{*}{27} & \multirow[t]{3}{*}{27} & \multirow[t]{3}{*}{-7.08} \\
\hline Inferior Frontal Gyrus & & & & & & & \\
\hline Superior Frontal Gyrus & & & & & & & \\
\hline Precentral Gyrus & 9 & $\mathrm{R}$ & 325 & 24 & 6 & 27 & -6.76 \\
\hline \multicolumn{8}{|l|}{ Inferior Frontal Gyrus } \\
\hline Precentral Gyrus & 6 & $\mathrm{R}$ & 54 & 66 & -3 & 27 & -5.03 \\
\hline Supramarginal Gyrus & 40 & $\mathrm{~L}$ & 345 & -54 & -48 & 36 & -7.07 \\
\hline \multicolumn{8}{|l|}{ Inferior Parietal Lobule } \\
\hline \multicolumn{8}{|l|}{ Postcentral Gyrus } \\
\hline Superior Parietal Lobule & 19 & $L$ & 527 & -30 & -72 & 48 & -6.15 \\
\hline \multicolumn{8}{|l|}{ Precuneus } \\
\hline Superior Frontal Gyrus & 8 & $\mathrm{R}$ & 59 & 24 & 12 & 51 & -5.49 \\
\hline Superior Frontal Gyrus & 6 & $\mathrm{~L}$ & 55 & -3 & 18 & 66 & -5.89 \\
\hline
\end{tabular}

The anatomical locations, approximate Brodmann areas (BA) and MNI coordinates that corresponded to the $t$-values of the representative peaks within each cluster were reported. A negative t-value means that fALFF was lower in this brain region when the AIS value is higher. All regions reached a voxel-level significance threshold of $P<0.001$, FWE correct $P=0.05$, cluster size $>30$ 
Table 7 The altered fALFF in brain regions of S-Acu group after 5-weeks acupuncture

\begin{tabular}{|c|c|c|c|c|c|c|c|}
\hline \multirow[t]{2}{*}{ Brain regions } & \multirow[t]{2}{*}{$\mathrm{BA}$} & \multirow[t]{2}{*}{ Side } & \multirow{2}{*}{$\begin{array}{l}\text { Cluster } \\
\text { size }\end{array}$} & \multicolumn{3}{|l|}{$\mathrm{MNI}$} & \multirow[t]{2}{*}{ t-value } \\
\hline & & & & $x$ & Y & Z & \\
\hline Cerebellum Posterior Lobe & & L & 94 & -15 & -78 & -51 & 5.75 \\
\hline Cerebellum Posterior Lobe & & $\mathrm{R}$ & 40 & 6 & -63 & -54 & 5.30 \\
\hline Cerebellum Posterior Lobe & & $\mathrm{R}$ & 30 & 42 & -66 & -54 & 5.09 \\
\hline Cerebellum Posterior Lobe & & $\mathrm{R}$ & 49 & 6 & -54 & -39 & 5.25 \\
\hline Brainstem & & $\mathrm{R}$ & 117 & 18 & -18 & -39 & 6.46 \\
\hline Brainstem & & $\mathrm{L}$ & 30 & -6 & -15 & -18 & 5.55 \\
\hline Inferior Temporal Gyrus & 20 & $\mathrm{R}$ & 33 & 60 & -24 & -21 & 5.22 \\
\hline Middle Temporal Gyrus & & L & 40 & -66 & -45 & -18 & 7.72 \\
\hline Superior Temporal Gyrus & 39 & L & 47 & -57 & -57 & 21 & 6.02 \\
\hline Parahippocampa Gyrus & 30 & $\mathrm{R}$ & 42 & 15 & -33 & -6 & 5.52 \\
\hline Precuneus & 31 & L & 42 & -3 & -51 & 30 & 4.56 \\
\hline Superior Frontal Gyrus & 6 & L & 38 & -12 & 9 & 72 & 6.59 \\
\hline Superior Frontal Gyrus & 6 & $\mathrm{R}$ & 30 & 9 & 0 & 72 & 6.00 \\
\hline
\end{tabular}

The anatomical locations, approximate Brodmann areas (BA) and MNI coordinates that corresponded to the $t$-values of the representative peaks within each cluster were reported. A positive $t$-value means that AALFF was increased in this brain region after acupuncture treatment. All regions reached a voxel-level significance threshold of $P<0.001$, FWE correct $P=0.05$, cluster size $>30$

\section{The altered fALFF regions of PI patients after acupuncture treatment}

The fALFF value of PI patients (S-Acu group: $n=15$; MAcu group: $\mathrm{n}=15$; N-Acu group: $\mathrm{n}=15$ ) were all changed after the 5-week acupuncture treatment. In S-Acu group, the fALFF of PI patients increased in bilateral cerebellum posterior lobe, bilateral brainstem, temporal gyrus (right inferior temporal gyrus, left middle temporal gyrus, left superior temporal gyrus), right parahippocampa gyrus, left precuneus, bilateral superior frontal gyrus $(P<0.001$, FWE correct $P=0.05$, cluster size $>30)$ (Table 7, Fig. 2). In M-Acu group, the fALFF of PI patients increased in right cerebellum posterior lobe, left parahippocampa gyrus, temporal gyrus (bilateral middle temporal gyrus, bilateral superior temporal gyrus), frontal gyrus (left middle frontal gyrus, left inferior frontal gyrus), parietal gyrus (bilateral precentral gyrus, bilateral postcentral gyrus, left inferior parietal lobule, left superior parietal lobule, left angular gyrus, left supramarginal gyrus, bilateral precuneus), left cuneus $(P<0.001$, FWE correct $P=0.05$, cluster size $>30)$ (Table 8, Fig. 3). In N-Acu group, the fALFF of PI patients increased in frontal gyrus (bilateral superior frontal gyrus, right medial frontal gyrus, bilateral middle frontal gyrus, right inferior frontal gyrus), right anterior cingulate, left insula $(P<0.001$, FWE correct $P=0.05$, cluster size $>30$ ) (Table 9, Fig. 4). We also listed the regions where fALFF were negative correlation with AIS scores, and the regions in which could be regulated by three acupuncture regimens (Fig. 5). The results showed that the brain regions where fALFF were negative correlation with AIS scores could be partially regulated in each group, and the $\mathrm{M}$-Acu group could regulate more brain regions.

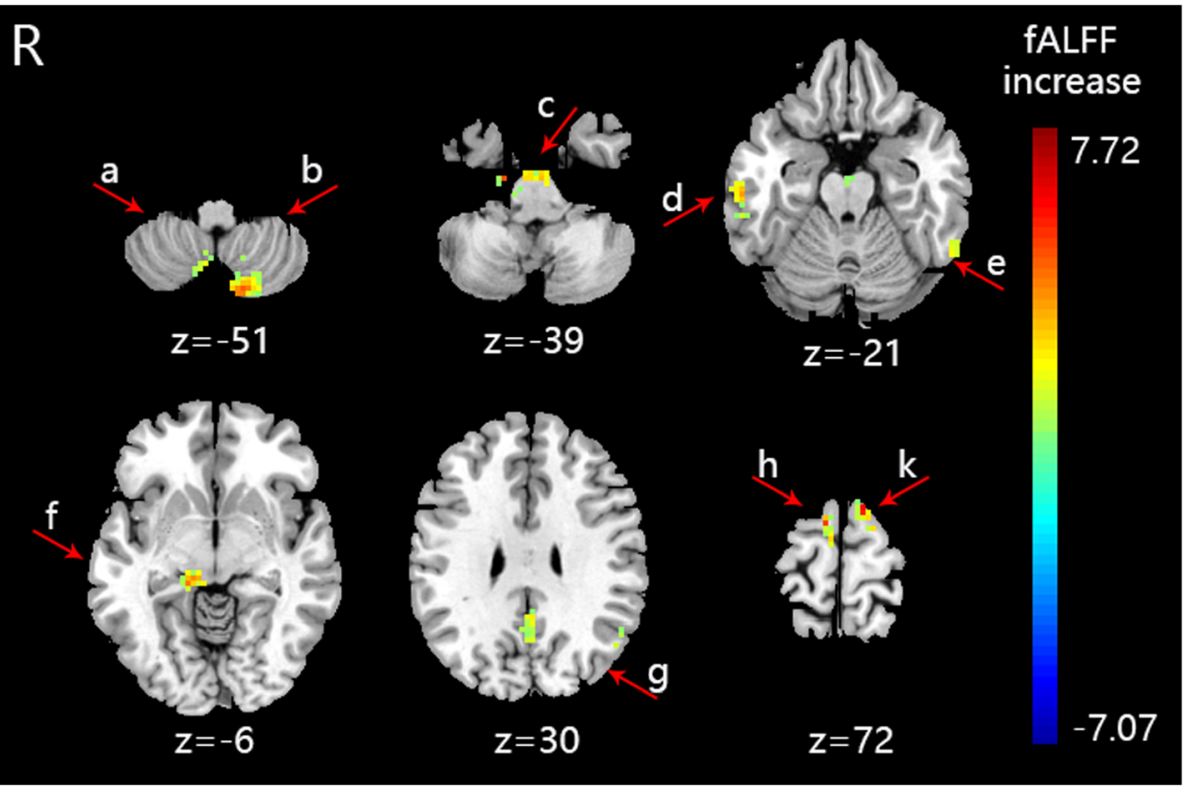

Fig. 2 The brain regions with altered fALFF of S-Acu group after acupuncture treatment $(P<0.001$, FWE correct $P=0.05$, cluster size $>30)$. R: right brain. a: right cerebellum posterior lobe, $\mathbf{b}$ : left cerebellum posterior lobe, $\mathbf{c}$ : brainstem, $\mathbf{d}$ : right inferior temporal gyrus, e: left middle temporal gyrus, f: right parahippocampa gyrus, g: left precuneus, h: right superior frontal gyrus, $\mathbf{k}$ : left superior frontal gyrus. Colorbar refer to the increased fALFF value 
Table 8 The altered fALFF in brain regions of M-Acu group after 5-weeks acupuncture

\begin{tabular}{|c|c|c|c|c|c|c|c|}
\hline \multirow[t]{2}{*}{ Brain regions } & \multirow[t]{2}{*}{$\mathrm{BA}$} & \multirow[t]{2}{*}{ Side } & \multirow{2}{*}{$\begin{array}{l}\text { Cluster } \\
\text { size }\end{array}$} & \multicolumn{3}{|l|}{$\mathrm{MNI}$} & \multirow[t]{2}{*}{ t-value } \\
\hline & & & & $x$ & Y & Z & \\
\hline Cerebellum Posterior Lobe & & $R$ & 50 & 15 & -33 & -60 & 5.03 \\
\hline Parahippocampa Gyrus & 36 & L & 38 & -27 & -36 & -15 & 5.70 \\
\hline Middle Temporal Gyrus & $21 / 22$ & L & 261 & -54 & -57 & 0 & 6.82 \\
\hline \multicolumn{8}{|l|}{ Superior Temporal Gyrus } \\
\hline Superior Temporal Gyrus & & R & 33 & 45 & -18 & -9 & 4.96 \\
\hline Middle Temporal Gyrus & 37 & $\mathrm{R}$ & 54 & 48 & -39 & -9 & 6.12 \\
\hline Superior Temporal Gyrus & & $\mathrm{R}$ & 73 & 45 & -39 & 9 & 6.67 \\
\hline Middle Temporal Gyrus & & L & 42 & -45 & -78 & 9 & 5.09 \\
\hline Middle Frontal Gyrus & & L & 119 & -39 & 30 & 24 & 5.69 \\
\hline Inferior Frontal Gyrus & $6 / 9$ & L & 525 & -45 & 3 & 30 & 7.60 \\
\hline \multicolumn{8}{|l|}{ Precentral Gyrus } \\
\hline \multicolumn{8}{|l|}{ Postcentral Gyrus } \\
\hline Precentral Gyrus & & R & 43 & 42 & -3 & 30 & 4.93 \\
\hline Postcentral Gyrus & $3 / 4$ & $\mathrm{R}$ & 66 & 60 & -21 & 39 & 5.64 \\
\hline \multicolumn{8}{|l|}{ Precentral Gyrus } \\
\hline Inferior Parietal Lobule & 40 & L & 371 & -48 & -57 & 33 & 6.00 \\
\hline \multicolumn{8}{|l|}{ Angular Gyrus } \\
\hline \multicolumn{8}{|l|}{ Supramarginal Gyrus } \\
\hline \multicolumn{8}{|l|}{ Postcentral Gyrus } \\
\hline Cuneus & $18 / 19$ & L & 370 & -21 & -51 & 39 & 5.65 \\
\hline \multicolumn{8}{|l|}{ Precuneus } \\
\hline \multicolumn{8}{|l|}{ Superior Parietal Lobule } \\
\hline Precuneus & 39 & $\mathrm{R}$ & 67 & 33 & -72 & 42 & 5.02 \\
\hline
\end{tabular}

The anatomical locations, approximate Brodmann areas (BA) and MNI coordinates that corresponded to the $\mathrm{t}$-values of the representative peaks within each cluster were reported. A positive $t$-value means that fALFF was increased in this brain region after acupuncture treatment. All regions reached a voxel-level significance threshold of $P<0.001$, FWE correct $P=0.05$, cluster size $>30$

The different altered fALFF regions of PI patients in different groups

Compared the increased fALFF in brain regions between the S-Acu group and N-Acu group, we found the increased fALFF in right anterior cingulate and left insula of N-Acu group were higher than that of S-Acu group. The increased fALFF in left cerebellum posterior lobe and right brainstem of S-Acu group were higher than that of N-Acu group $(P<0.005$, FWE correct $P=0.05$, cluster size $>30$ ) (Table 10).

Compared the increased fALFF in brain regions between the S-Acu group and M-Acu group, we found the increased fALFF in left parahippocampa gyrus, bilateral superior temporal gyrus, left middle temporal gyrus, right lentiform nucleus, left middle frontal gyrus, left inferior frontal gyrus, left precentral gyrus, left postcentral gyrus, left inferior parietal lobule, left superior parietal lobule, left anterior cingulate, left supramarginal gyrus, left angular gyrus and right precuneus of M-Acu group were higher than those of S-
Acu group $(P<0.005$, FWE correct $P=0.05$, cluster size $>30$ ) (Table 10).

\section{Discussion}

According to the Traditional Chinese Medicine (TCM) theory, acupuncture play a therapeutic role by stimulating at the acupoints, and producing special needling/ tactile somatosensory which is called De-Qi. Patients experienced De-Qi as multiple unique sensations at the needle site and surrounding regions. MASS scale was used as an appropriate method to measure De-Qi sensation [26]. In our study, there was no significant difference of the MASS scale among three groups.

However, not limited to this, the placebo effect also plays a role in treatment process. Research suggested that the sham acupuncture without somatosensory tactile stimulation could still improve the clinical symptoms [27]. The primary outcomes of our study suggested that clinical symptoms were improved in all groups after 5 weeks acupuncture treatment, including the N-Acu 


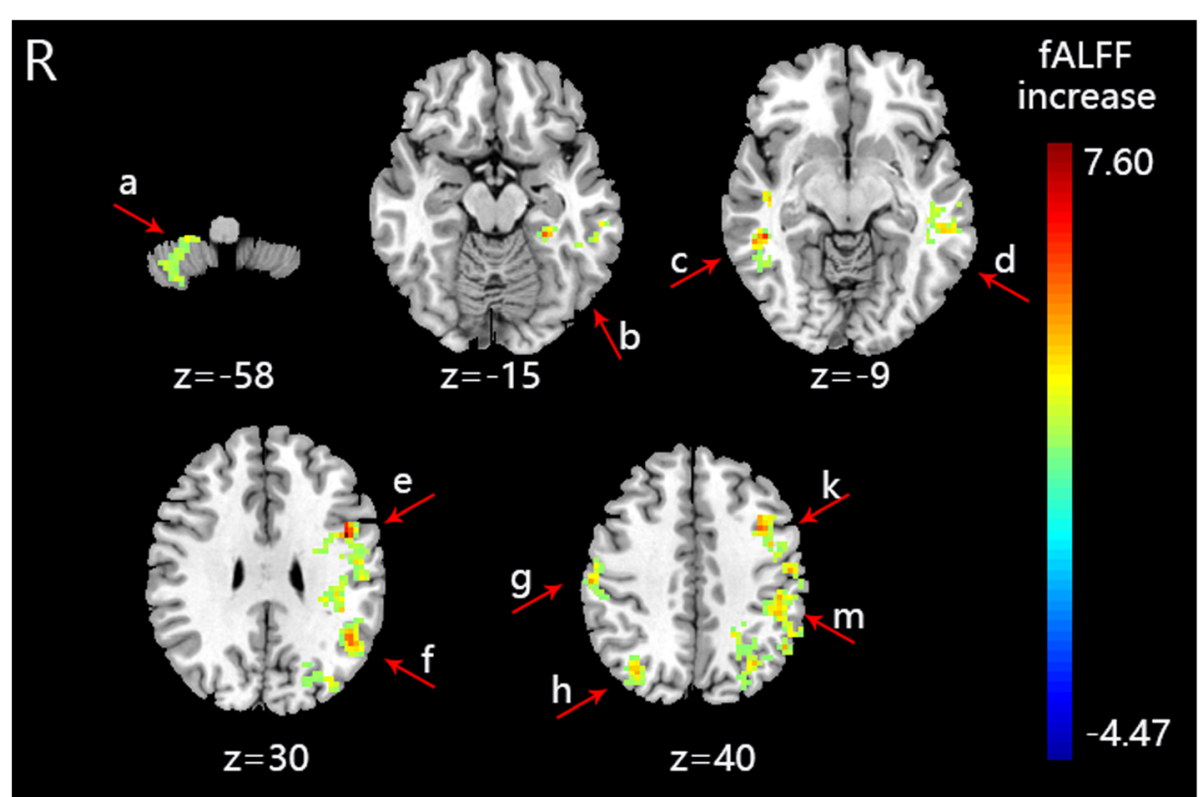

Fig. 3 The brain regions with altered fALFF of M-Acu group after acupuncture treatment $(P<0.001$, FWE correct $P=0.05$, cluster size $>30)$. R: right brain. a: right cerebellum posterior lobe, $\mathbf{b}$ : left parahippocampa gyrus, $\mathbf{c}$ : left superior temporal gyrus and middle temporal gyrus, $\mathbf{d}$ : right superior temporal gyrus and middle temporal gyrus, e: left inferior frontal gyrus, $\mathbf{f}$ : left supramarginal gyrus, $\mathbf{g}$ : right precentral gyrus, $\mathbf{h}$ : right precuneus, $\mathbf{k}$ : left middle frontal gyrus, m: left postcentral gyrus. Colorbar refer to the increased fALFF value

group (sham point). However, the decreased PSQI score of S-Acu was higher than that of N-Acu group. In addition, the PSG results suggested that the TST $(P=$ $0.020)$ and $\mathrm{R}(P=0.001)$ of $\mathrm{S}$-Acu group were significant increased after 5-weeks acupuncture. Although the TST and $\mathrm{R}$ were also increased in $\mathrm{N}$-Acu group, no significant difference was found after 5-weeks acupuncture. Therefore, we suggested that acupuncture could improve the symptoms and patient's subjective experience, and the real acupoints were more effective than sham points.

Table 9 The altered fALFF in brain regions of N-Acu group after 5-weeks acupuncture

\begin{tabular}{|c|c|c|c|c|c|c|c|}
\hline \multirow[t]{2}{*}{ Brain regions } & \multirow[t]{2}{*}{ BA } & \multirow[t]{2}{*}{ Side } & \multirow{2}{*}{$\begin{array}{l}\text { Cluster } \\
\text { size }\end{array}$} & \multicolumn{3}{|l|}{$\mathrm{MNI}$} & \multirow[t]{2}{*}{ t-value } \\
\hline & & & & $x$ & $\mathrm{Y}$ & $\bar{Z}$ & \\
\hline Superior Frontal Gyrus & & $\mathrm{L}$ & 34 & -21 & 63 & 3 & 4.63 \\
\hline Superior Frontal Gyrus & 10 & $\mathrm{R}$ & 364 & 21 & 60 & 6 & 5.38 \\
\hline \multicolumn{8}{|l|}{ Medial Frontal Gyrus } \\
\hline \multicolumn{8}{|l|}{ Anterior Cingulate } \\
\hline Insula & 13 & $L$ & 30 & -33 & 21 & 6 & 4.16 \\
\hline Middle Frontal Gyrus & 46 & $\mathrm{~L}$ & 33 & -45 & 36 & 21 & 4.49 \\
\hline Middle Frontal Gyrus & 46 & $\mathrm{R}$ & 83 & 42 & 24 & 24 & 4.44 \\
\hline Inferior Frontal Gyrus & 9 & $\mathrm{R}$ & 51 & 57 & 6 & 36 & 5.09 \\
\hline
\end{tabular}

The anatomical locations, approximate Brodmann areas (BA) and MNI coordinates that corresponded to the $t$-values of the representative peaks within each cluster were reported. A positive $t$-value means that fALFF was increased in this brain region after acupuncture treatment. All regions reached a voxel-level significance threshold of $P<0.001$, FWE correct $P=0.05$, cluster size $>30$
Then the efficacy of the two acupuncture regimens, single acupoint (HT-7) and combination of multiacupoints (HT-7, SP-6 and GV-20) were compared, the primary outcomes suggested that the decreased AIS score and PSQI score of M-Acu group were slightly higher than those of S-Acu group, although there was no significant difference between the two groups. In addition, the PSG results revealed that the TST and REM in S-Acu group were significantly increased. And in M-Acu group, the TST, SE, N3 and REM were significantly increased, while the AI and WASO were significantly decreased. REM sleep engage brain development functions to facilitate the formation and consolidation of certain types of memory and learning processes [28]. The prolongation of the REM stage is evidence that support the acupuncture at a single acupoint or a combination of multi-acupoints both have a positive effect on PI patients. Furthermore, the N3 time was prolonged in $\mathrm{M}$-Acu group, which was not observed in S-Acu group. And the increased TST of M-Acu group was significantly longer than that of S-Acu group. Above all, these findings of primary and secondary clinical outcomes suggested that the combination of multi-acupoints might enhance clinical efficacy.

In order to further explore the mechanism of acupuncture therapy, we designed the resting state fMRI study to observe the brain activity of PI patients before and after 5-weeks acupuncture. First, we studied the relationship between the brain activity (fALFF value) and 


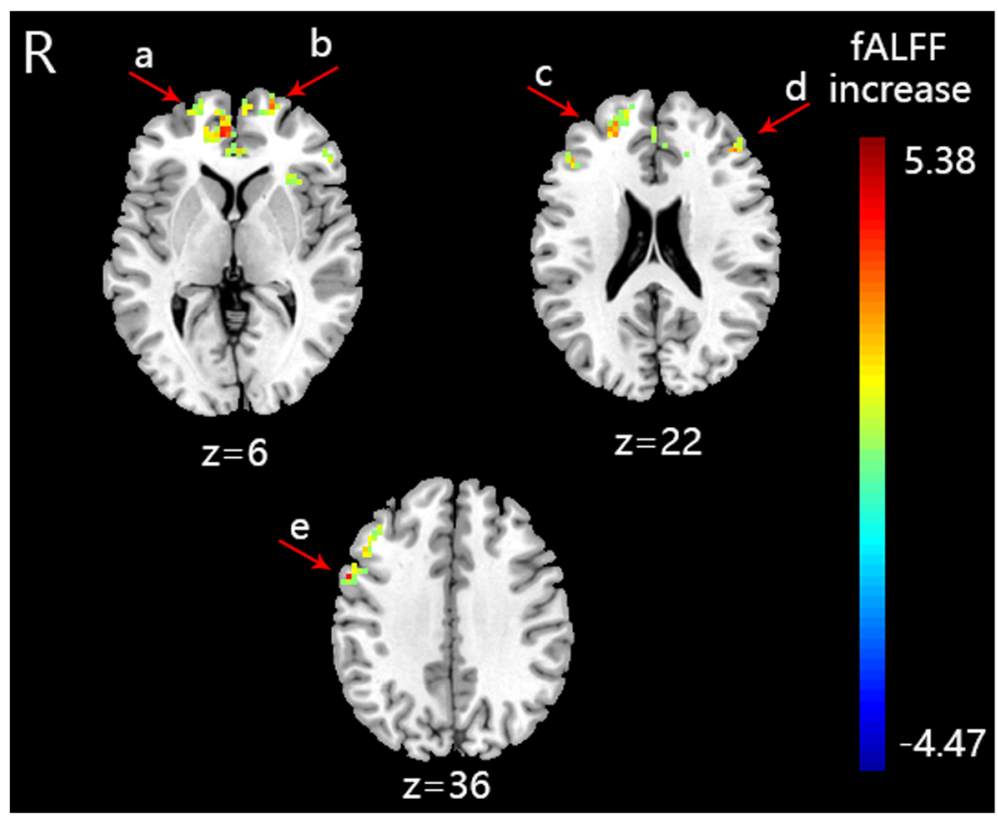

Fig. 4 The brain regions with altered fALFF of N-Acu group after acupuncture treatment $(P<0.001$, FWE correct $P=0.05$, cluster size $>30)$. R: right brain. a: right superior frontal gyrus and medial frontal gyrus, $\mathbf{b}$ : left superior frontal gyrus, c: right middle frontal gyrus, d: left middle frontal gyrus, e: right inferior frontal gyrus. Colorbar refer to the increased fALFF value

sleep quality (AIS score), we found that some brain regions had higher fALFF levels when AIS scores were lower. These regions included the right cerebellum posterior lobe $[29,30]$, temporal gyrus (left middle temporal gyrus [30], left superior temporal gyrus [15]), left extranuclear, right anterior cingulate [31], frontal gyrus (bilateral inferior frontal gyrus [30], left middle frontal gyrus $[29,30]$, bilateral superior frontal gyrus [29]), parietal lobule (right precentral gyrus, left supramarginal gyrus, left inferior parietal lobule [29,30], left superior parietal lobule, left precuneus [29, 31]). Researches suggested that differences in glucose metabolism rates or ALFF between insomnia patients and healthy controls in these brain regions [29-31]. Furthermore, our results suggested that acupuncture could modulate the ALFF value of these regions. And the number of regulated brain

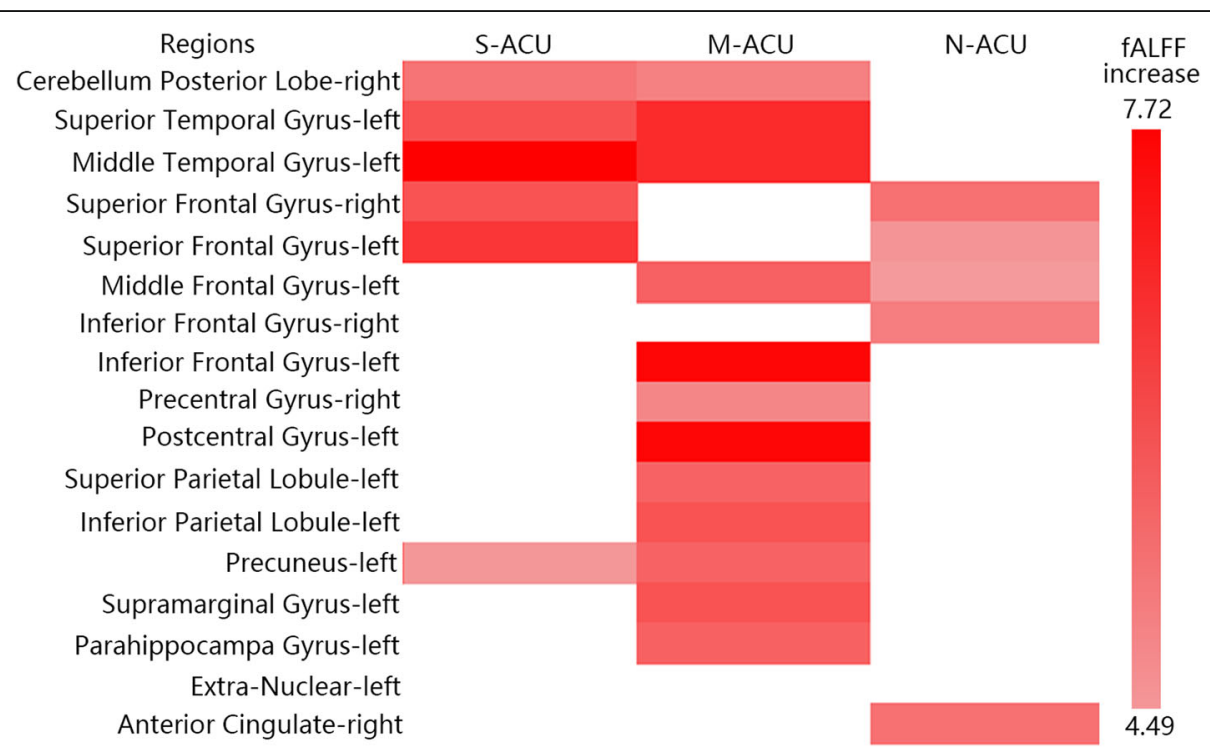

Fig. 5 The distribution of brain regions where fALFF were negative correlation with AIS scores, and brain regions which were regulated by three acupuncture regimens. Colorbar refer to the increased fALFF value 
Table 10 The different altered fALFF regions of different groups after acupuncture treatment

\begin{tabular}{|c|c|c|c|c|c|c|c|c|}
\hline \multirow[t]{2}{*}{ Group } & \multirow[t]{2}{*}{ Brain regions } & \multirow[t]{2}{*}{ BA } & \multirow[t]{2}{*}{ Side } & \multirow{2}{*}{$\begin{array}{l}\text { Cluster } \\
\text { size }\end{array}$} & \multicolumn{3}{|l|}{$\mathrm{MNI}$} & \multirow[t]{2}{*}{ t-value } \\
\hline & & & & & $\bar{x}$ & $\mathrm{Y}$ & Z & \\
\hline \multirow[t]{4}{*}{ S vs N } & Anterior Cingulate & 25 & $\mathrm{R}$ & 51 & 3 & 18 & -9 & -5.12 \\
\hline & Insula & & L & 168 & -15 & 30 & 15 & -6.93 \\
\hline & Cerebellum Posterior Lobe & & L & 84 & -15 & -57 & -57 & 4.51 \\
\hline & Brainstem & & $\mathrm{R}$ & 38 & 6 & -24 & -39 & 4.56 \\
\hline \multirow[t]{17}{*}{ S vs M } & Parahippocampa Gyrus & & L & 35 & -27 & -36 & -15 & -5.18 \\
\hline & Superior Temporal Gyrus Middle Temporal Gyrus & 22 & L & 348 & -57 & -18 & -3 & -5.83 \\
\hline & Superior Temporal Gyrus & & $\mathrm{R}$ & 31 & 45 & -39 & 9 & -5.02 \\
\hline & Lentiform Nucleus & & $\mathrm{R}$ & 91 & 18 & 9 & 0 & -4.92 \\
\hline & Middle Frontal Gyrus & & L & 37 & -30 & 42 & 18 & -5.09 \\
\hline & Middle Frontal Gyrus & & L & 33 & -36 & 15 & 42 & -4.69 \\
\hline & Middle Frontal Gyrus & 6 & L & 50 & -27 & 0 & 66 & -4.66 \\
\hline & Precentral Gyrus & & L & 126 & -51 & 0 & 12 & -5.40 \\
\hline & Inferior Frontal Gyrus & & & & & & & \\
\hline & Inferior Frontal Gyrus & 9 & L & 46 & -48 & 3 & 27 & -5.58 \\
\hline & Postcentral Gyrus & 40 & L & 152 & -48 & -27 & 39 & -4.61 \\
\hline & Inferior Parietal Lobule & & & & & & & \\
\hline & Superior Parietal Lobule & 7 & L & 55 & -33 & -69 & 48 & -5.09 \\
\hline & Anterior Cingulate & & L & 62 & -24 & 27 & 24 & -5.32 \\
\hline & Supramarginal Gyrus & 40 & $L$ & 110 & -54 & -48 & 36 & -5.73 \\
\hline & Angular Gyrus & & & & & & & \\
\hline & Precuneus & 39 & $\mathrm{R}$ & 33 & 36 & -69 & 42 & -4.59 \\
\hline
\end{tabular}

The anatomical locations, approximate Brodmann areas (BA) and MNI coordinates that corresponded to the t-values of the representative peaks within each cluster were reported. S vs N S-Acu group vs N-Acu group. A positive t-value means that the increased fALFF in this brain region of S-Acu group was higher than that of N-Acu group. S vs M S-Acu group vs M-Acu group. A negative t-value means that the increased fALFF in this brain region of S-Acu group was lower than that of M-Acu group. All regions reached a voxel-level significance threshold of $P<0.005$, FWE correct $P=0.05$, cluster size $>30$

areas in M-Acu group is much higher than that in SAcu group or N-Acu group.

Compared with the S-Acu group, the increased fALFF of the $\mathrm{N}$-Acu group in anterior cingulate and insula were more obvious. Studies have shown that real acupuncture could exhibit greater activation in the anterior cingulate gyrus and insula regions than phantom acupuncture without somatosensory tactile stimulation [27]. However, sham acupuncture in our trial would produce pain stimulation, and MASS results showed that sharp pain scores were slightly higher than that in the S-Acu group, although there was no statistical difference between the two groups. Therefore, we speculated that the increased fALFF in the anterior cingulate gyrus and insula may be caused by pain stimulation. And the increased fALFF in the cerebellum posterior lobe and brainstem may be caused by the stimulating on specific acupoint of S-Acu group.

Compared with the S-Acu group, we found the increased fALFF in parahippocampa gyrus, superior temporal gyrus, middle temporal gyrus, lentiform nucleus, middle frontal gyrus, inferior frontal gyrus, precentral gyrus, postcentral gyrus, inferior parietal lobule, superior parietal lobule, anterior cingulate, supramarginal gyrus, angular gyrus and precuneus of $\mathrm{M}$-Acu group were more obvious.

The brain activity of some brain regions which were regulated in M-Acu group, including superior temporal gyrus [15], middle temporal gyrus [30], middle frontal gyrus [29, 30], inferior frontal gyrus [30], superior parietal lobule, inferior parietal lobule [29, 30], anterior cingulate [31], supramarginal gyrus, precuneus [29, 31], were varied between insomnia patients and healthy controls. And our study also suggested that brain activity in these brain regions were negatively correlated with AIS scores. Therefore, we considered that for primary insomnia, multi-acpoints stimulation might produce a greater therapeutic effect.

\section{Conclusion}

In our clinical trials, acupuncture has been proven to be beneficial for PI patients, and the combination of multiacupoints could improve its efficacy. The relationship between the brain activity (fALFF value) and sleep 
quality (AIS score) suggested that the brain activity of certain regions was higher when sleep quality was better, while part of these regions could be regulated by acupuncture on single acupoints/sham point/combination of multi-acupoints. The effect of multi-acupoints group on regulating the brain activity was most obvious, which may be the possible mechanism of that multi-acupoints stimulating could improve the acupuncture efficacy.

\section{Limitations}

The present trial aims to select a more effective acupoint scheme for insomnia in clinical acupuncture treatment. The different efficacy between a single acupoint and a combination of acupoints were mainly compared in the present trial. However, the present data remains preliminary, and the comparison of efficacy produced by these different single acupoints were based on the previous studies, which have not been investigated in the present trial. More studies are needed to compare the efficacy difference among different single acupoints.

\begin{abstract}
Abbreviations
ACC: Anterior cingulate cortex; Al: Arousal index; AIS: Athens insomnia scale; ALFF: Amplitude of low-frequency fluctuation; BA: Brodmann area; BOLD: Blood oxygenation level dependent; DPARSF: Data processing assistant for resting-state fMRI; FA: Flip angle; FOV: Field of view; FWE: Family wise error rate; MASS: Massachusetts General Hospital acupuncture sensation scales; MNI: Montreal neurological institute; NREM: Non-rapid eye movement; PI: Primary insomnia; PSG: Polysomnography; PSQI: Pittsburgh sleep quality index; REM: Rapid eye movement; Rs-fMRI: Resting state functional magnetic resonance imaging; SAS: Self-rating anxiety scale; SDS: Self-rating depression scale; SE: Sleep efficient; SOL: Sleep onset latency; SPM: Statistical parametric mapping toolbox; TR: Repetition time; TE: Echo time; TST: Total sleep time; WASO: Wake after sleep onset
\end{abstract}

\section{Acknowledgements}

Not Applicable.

\begin{abstract}
Authors' contributions
YW: The analysis and interpretation of clinical data and fMRI data, drafting the manuscript and substantively revised it. TL and LH: the acquisition and analysis of clinical data. ZL: the acquisition and analysis of fMRI data. FW and ZW: Conception and design of the study. JM and ZX: Conception and design of the study, provided decisive feedback and suggestions to improve the manuscript. All authors agreed to be personally accountable for the author's own contributions and to ensure that questions related to the accuracy or integrity of any part of the work, and have read the final manuscript and approved the submitted version.
\end{abstract}

\section{Funding}

This work was full financial supported by the National Basic Research Program (Grant 973) of China (2014CB543100) in China-Japan Union Hospital of Jilin University and Changchun University of Chinese Medicine.

\section{Availability of data and materials}

The datasets generated and/or analyzed in the present study can be available from the corresponding author upon reasonable request.

\section{Ethics approval and consent to participate}

All procedures performed in studies involving human participants were in accordance with the ethical standards of the institutional and/or national research committee and with the 1964 Helsinki declaration and its later amendments or comparable ethical standards. All methodologies were approved by the Ethics Committee of Changchun University of Chinese
Medicine (Reference: CCZYFYLL2014-043). A written signed informed consent was provided by all individual participants included in the study.

Consent for publication

Not Applicable.

\section{Competing interests}

Yu-Kai Wang declares that she has no conflict of interest. Tie Li declares that he has no conflict of interest. Li-Juan Ha declares that she has no conflict of interest. Zhong-Wen Lv declares that he has no conflict of interest. Fu-Chun Wang declares that he has no conflict of interest. Zhi-Hong Wan declares that he has no conflict of interest. Jing Mang declares that he has no conflict of interest. Zhong-Xin Xu declares that he has no conflict of interest.

\section{Author details}

'Department of Neurology, China-Japan Union Hospital of Jilin University, Changchun, Jilin 130033, P.R. China. ${ }^{2}$ Department of Acupuncture and Moxibustion, Changchun University of Chinese Medicine, Changchun, Jilin 130117, P.R. China. ${ }^{3}$ Department of Radiology, China-Japan Union Hospital of Jilin University, Changchun, Jilin 130033, P.R. China. ${ }^{4}$ The '973' National Basic Research Program of China, Changchun University of Chinese Medicine, Changchun, Jilin 130117, P.R. China.

Received: 29 November 2019 Accepted: 24 May 2020

Published online: 17 August 2020

\section{References}

1. Buysse DJ. Insomnia. JAMA. 2013;309:706-16. https://doi.org/10.1001/jama. 2013.193 URL: https://www.ncbi.nlm.nih.gov/pmc/articles/PMC3632369/.

2. Kessler RC, Berglund PA, Coulouvrat $C$, et al. Insomnia and the performance of US workers: results from the America insomnia survey. Sleep. 2011;34(9): 1161-71. https://doi.org/10.5665/SLEEP.1230 URL: https://www.ncbi.nlm.nih. gov/pmc/articles/PMC3157657/.

3. Irwin MR. Why sleep is important for health: a psychoneuroimmunology perspective. Annu Rev Psychol. 2015;66:143-72. https://doi.org/10.1146/ annurev-psych-010213-115205 URL: https://www.ncbi.n/m.nih.gov/pmc/ articles/PMC4961463/.

4. Lai MM, Lin CC, Lin CC, Liu CS, Li TC, Kao CH. Long-term use of zolpidem increases the risk of major injury: a population-based cohort study. Mayo Clin Proc. 2014;89:589-94. https://doi.org/10.1016/j.mayocp.2014.01.021 URL: https://www.mayoclinicproceedings.org/article/S0025-6196(14)00106-2/ fulltext.

5. Guo J, Wang LP, Liu CZ, et al. Efficacy of acupuncture for primary insomnia: a randomized controlled clinical trial. Evid Based Complement Alternat Med. 2013;2013:163850. https://doi.org/10.1155/2013/163850 URL: https://www. ncbi.nlm.nih.gov/pmc/articles/PMC3789397/.

6. Shergis $\mathrm{J}, \mathrm{Ni} \mathrm{X}$, Jackson $\mathrm{ML}$, et al. A systematic review of acupuncture for sleep quality in people with insomnia. Complement Ther Med. 2016;26:1120. https://doi.org/10.1016/j.ctim.2016.02.007 URL: https://www.ncbi.nlm.nih. gov/pubmed/27261976.

7. Wang H, Yang G, Wang S, Zheng X, Zhang W, Li Y. The Most commonly treated acupuncture indications in the United States: a cross-sectional study. Am J Chin Med. 2018;9:1-33. https://doi.org/10.1142/S0192415X18500738 URL: https://www.worldscientific.com/doi/abs/10.1142/S0192415X18500738.

8. Fang J, Jin Z, Wang $Y$, et al. The salient characteristics of the central effects of acupuncture needling: limbic-Paralimbic-neocortical network modulation. Hum Brain Mapp. 2009;30:1196-206. https://doi.org/10.1002/hbm.20583 URL: https://onlinelibrary.wiley.com/doi/abs/10.1002/hbm.20583.

9. Huang $W$, Kutner N, Bliwise DL. Autonomic activation in insomnia: the case for acupuncture. J Clin Sleep Med. 2011;7:95-102 URL: https://www.ncbi. nlm.nih.gov/pmc/articles/PMC3041619/.

10. Napadow V, Lee J, Kim J, et al. Brain correlates of phasic autonomic response to acupuncture stimulation: an event-related fMRI study. Hum Brain Mapp. 2013;34:2592-606. https://doi.org/10.1002/hbm.22091 URL: https://www.ncbi.nlm.nih.gov/pmc/articles/PMC3646924/.

11. Zheng Y, Qu S, Wang N, et al. Post-stimulation effect of electroacupuncture at Yintang (EX-HN3) and GV20 on cerebral functional regions in healthy volunteers: a resting functional MRI study. Acupunct Med. 2012;30:307-15. https://doi.org/10.1136/acupmed-2011-010123 URL: https://journals.sagepub. com/doi/abs/10.1136/acupmed-2011-010123. 
12. Chen JE, Glover GH. Functional magnetic resonance imaging methods. Neuropsychol Rev. 2015;25(3):289-313. https://doi.org/10.1007/s11065-0159294-9 URL: https://www-ncbi-nlm-nih-gov.ermg.vghtc.gov.tw/pmc/articles/ PMC4565730/.

13. Fransson P. Spontaneous low-frequency BOLD signal fluctuations: an fMRI investigation of the resting-state default mode of brain function hypothesis. Hum Brain Mapp. 2005;26(1):15-29. https://doi.org/10.1002/hbm.20113 URL: https://www.ncbi.nlm.nih.gov/pubmed/15852468.

14. Zou QH, Zhu CZ, Yang Y, et al. An improved approach to detection of amplitude of low-frequency fluctuation (ALFF) for resting-state fMRI: fractional ALFF. J Neurosci Methods. 2008;172:137-41. https://doi.org/10. 1016/j.jneumeth.2008.04.012 URL: https://www-ncbi-nlm-nih-gov.ermg. vghtc.gov.tw/pmc/articles/PMC3902859/.

15. Wang YK, Shi XH, Wang YY, et al. Evaluation of the age-related and genderrelated differences in patients with primary insomnia by fractional amplitude of low-frequency fluctuation: A resting-state functional magnetic resonance imaging study. Medicine (Baltimore). 2020;99(3):e18786. https:// doi.org/10.1097/MD.0000000000018786 URL:https://journals.lww.com/mdjournal/FullText/2020/01170/Evaluation_of_the_age_related_and_gender_ related.46.aspx.

16. Zhao K. Acupuncture for the treatment of insomnia. Int Rev Neurobiol. 2013;111:217-34. https://doi.org/10.1016/B978-0-12-411545-3.00011-0 URL: https://www.ncbi.nlm.nih.gov/pubmed/24215925.

17. Huang $K$, Liang $S, X u Y$, Lu S. Law of acupoint selection in acupuncture treatment for insomnia based on data mining method. Zhongguo Zhen Jiu. 2015;35(9):960-3. https://doi.org/10.13703/j.0255-2930.2018.07.030 Chinese. URL: https://www.ncbi.nlm.nih.gov/pubmed/26721161.

18. Wang, J., Wang, J., Wang, L., Zhang, Y. Senile insomnia treated with integrated acupuncture and medication therapy: a randomized controlled trial. Zhongguo Zhen Jiu. 2015;35(6):544-548. https://pubmed-ncbi-nlm-nihgov.cmich.idm.oclc.org/26480547/.

19. Zhan, J., Pan, R., Guo, Y., et al. Acupuncture at Baihui(GV 20) and Shenting (GV 24) combined with basic treatment and regular rehabilitation for post-stroke cognitive impairment:a randomized controlled trial. Zhongguo Zhen Jiu. 2016;36(8):803-806. https://doi.org/10.13703/j.02552930.2016.08.007. Chinese. https://www-ncbi-nlm-nih-gov.ermg.vghtc.gov. tw/pubmed/29231563.

20. Yeung WF, Chung KF, Zhang SP, Yap TG, Law AC. Electroacupuncture for primary insomnia: a randomized controlled trial. Sleep. 2009;32(8):1039-47. https://doi.org/10.1093/sleep/32.8.1039 URL: https://www.ncbi.nlm.nih.gov/ pubmed/19725255.

21. Takamoto K, Hori E, Urakawa S, et al. Cerebral hemodynamic responses induced by specific acupuncture sensations during needling at trigger points: a near-infrared spectroscopic study. Brain Topogr. 2010;23:279-91. https://doi.org/10.1007/s10548-010-0148-8 URL: https://link.springer.com/ article/10.1007\%2Fs10548-010-0148-8.

22. Soldatos CR, Dikeos DG, Paparrigopoulos TJ. Athens insomnia scale: validation of an instrument based on iCD-10 criteria. J Psychosom Res. 2000; 48:555-60. https://doi.org/10.1016/s0022-3999(00)00095-7 URL: https://www. ncbi.nlm.nih.gov/pubmed/11033374.

23. Buysse DJ, Reynolds CF, Monk TH, Berman SR, Kupfer DJ. The Pittsburgh sleep quality index: a new instrument for psychiatric practice and research. J Psychiatry Res. 1989;28:193-213. https://doi.org/10.1016/0165-

1781(89)90047-4 URL: https://linkinghub.elsevier.com/retrieve/pii/01651781(89)90047-4.

24. He $Y$, Wang $L$, Zang $Y$, et al. Regional coherence changes in the early stages of Alzheimer's disease: a combined structural and resting-state functional MRI study. Neuroimage. 2007;35:488-500. https://doi.org/10.1016/j. neuroimage.2006.11.042 URL: https://www.ncbi.nlm.nih.gov/pubmed/ 17254803.

25. Song $X W$, Dong $Z Y$, Long $X Y$, et al. REST: a toolkit for resting-state functional magnetic resonance imaging data processing. PLoS One. 2011;6: e25031. https://doi.org/10.1371/journal.pone.0025031 URL: https://wwwncbi-nlm-nih-gov.cmich.idm.oclc.org/pubmed/21949842.

26. Shi GX, Li QQ, Liu CZ, et al. Effect of acupuncture on Deqi traits and pain intensity in primary dysmenorrhea: analysis of data from a larger randomized controlled trial. BMC Complement Altern Med. 2014;14:69. https://doi.org/10.1186/1472-6882-14-69 URL: https://www-ncbi-nlm-nihgov.cmich.idm.oclc.org/pmc/articles/PMC3933504/.

27. Makary MM, Lee J, Lee E, et al. Phantom acupuncture induces placebo credibility and vicarious sensations: a parallel fMRI study of low Back pain patients. Sci Rep. 2018;8(1):930. https://doi.org/10.1038/s41598-017-18870-1 URL: https://www.ncbi.nlm.nih.gov/pmc/articles/PMC5772373/.

28. Li W, Ma L, Yang G, Gan WB. REM sleep selectively prunes and maintains new synapses in development and learning. Nat Neurosci. 2017;20(3):42737. https://doi.org/10.1038/nn.4479 URL: https://www.ncbi.nlm.nih.gov/pmc/ articles/PMC5535798/.

29. Zhou F, Huang S, Zhuang Y, Gao L, Gong H. Frequency-dependent changes in local intrinsic oscillations in chronic primary insomnia: a study of the amplitude of low-frequency fluctuations in the resting state. Neuroimage Clin. 2016;15:458-65. https://doi.org/10.1016/j.nicl.2016.05.011 URL: https:// www-ncbi-nlm-nih-gov.ermg.vghtc.gov.tw/pubmed/28649490.

30. Li C, Ma X, Dong M, et al. Abnormal spontaneous regional brain activity in primary insomnia: a resting-state functional magnetic resonance imaging study. Neuropsychiatr Dis Treat. 2016;12:1371-8. https://doi.org/10.2147/ NDT.S109633 URL: https://www-ncbi-nlm-nih-gov.ermg.vghtc.gov.tw/ pubmed/27366068.

31. Kay DB, Karim HT, Soehner AM, et al. Sleep-wake differences in relative regional cerebral metabolic rate for glucose among patients with insomnia compared with good sleepers. Sleep. 2016;39(10):1779-94. https://doi.org/ 10.5665/sleep.6154 URL: https://www-ncbi-nlm-nih-gov.ermg.vghtc.gov.tw/ pubmed/27568812.

\section{Publisher's Note}

Springer Nature remains neutral with regard to jurisdictional claims in published maps and institutional affiliations.
Ready to submit your research? Choose BMC and benefit from:

- fast, convenient online submission

- thorough peer review by experienced researchers in your field

- rapid publication on acceptance

- support for research data, including large and complex data types

- gold Open Access which fosters wider collaboration and increased citations

- maximum visibility for your research: over $100 \mathrm{M}$ website views per year

At BMC, research is always in progress.

Learn more biomedcentral.com/submissions 